\title{
BIDIRECTIONAL REFLECTANCE DISTRIBUTION FUNCTION (BRDF) OF MIXED PIXELS
}

\author{
Fadi Kizel and Yulia Vidro
}

Dept. of Mapping and Geoinformation Engineering, Technion-Israel Institute of Technology, Haifa, 32000.

fadikizel@technion.ac.il; yuliav@campus.technion.ac.il

KEY WORDS: Remote sensing, Spectral unmixing, BRDF, Hyperspectral imaging, Semi-empirical models, Linear mixture model.

\begin{abstract}
:
Hyperspectral imaging is crucial for a variety of land-cover mapping and analyzing tasks. The available large number of reflected light measurements along a wide range of wavelengths allows for distinguishing between different materials under various conditions. Though, several effects bear an undesired variability within hyperspectral images and increase the complexity of interpreting such data. Two of the most significant effects in this regard are the BRDF and the spectral mixture. Due to the first, the acquisitions geometrical and viewing conditions influences the measured spectral signature of a surface to a large extent. On the other hand, because of the typical low spatial resolution of remotely sensed images, each pixel can contain more than one material. Despite much research addressing either the BRDF effect and ways to correct it or the spectral unmixing, too few works considered these two effects' mutual influence. In this work, we study the BRDF of mixed pixels and present preliminary insights of testing a strategy to correct its undesired impact on the data by incorporating the EMs fractions within an unmixing-based semi-empirical correction model. Experimental results using real laboratory data acquired under controlled conditions clearly show the significant improvement of the corrected reflectance results through the proposed model.
\end{abstract}

\section{INTRODUCTION}

Spectral data allows for distinguishing between distinct materials based on their measured reflectance (Landgrebe, 2002; Shaw and Burke, 2003). However, besides a natural spectral variability among pixels, an undesired variation of these pixels' reflectance exists due to several influences. The Bidirectional Reflectance Distribution Function (BRDF) and the spectral mixture are two of the main effects bearing a challenge in analyzing spectral data. Due to the BRDF, the surface's measured spectral signature depends on the acquisition's geometrical and viewing conditions. On the other hand, because of the typical low spatial resolution of remotely sensed images, pixels containing more than one material. Regarding the BRDF effect, given an image acquired under a particular geometrical configuration (Roy et al., 2016), a pixel's reflectance still depends on its spatial location within the scene. This dependency negatively affects the results of essential remote sensing tasks, e.g., classification (Faran et al., 2019; Melgani and Bruzzone, 2004), spectral unmixing (Kizel and Benediktsson, 2020) (Keshava and Mustard, 2002), and change detection (Eismann et al., 2008).

Reducing this dependency is critical; therefore, a BRDF correction is usually applied to modulate the undesired trend on the data that occurs due to this effect and then subtract it from the measurements. For this purpose, three main approaches are usually adopted based on physical, empirical, and semiempirical models:

a) Physical BRDF models describe the interactions of the incident radiation with a specific target considering biophysical parameters (Walthall, 1997), (Roberts, 2001). These models are more complex and have many parameters, relatively to empirical or semi-empirical models.

b) Empirical models are not focused on explaining biophysical processes that affect BRDF but rather explain mathematically the observed bidirectional reflectance derived from multi-angular data sets (Roberts, 2009).

c) A semi-empirical model combines simplicity and ease of inversion of a linear empirical model using a combination of weighted kernels and retaining some physical meaning in its parameters (Disney and Lewis, 1998). Models of this type are often referred to as linear semi-empirical kernel-based (LiSK) BRDF models (Jiao et al., 2018; Roujean et al., 1992).

Many research works addressed the BRDF effect while proposing methodologies for reducing its negative influence on the data. Besides, plenty of research tackled the spectral mixture analysis and proposed algorithms for estimating the abundance fraction of each different material (landcover type), so-called endmember (EM), within the pixel. Nevertheless, too few works addressed the mutual influence of these two effects. On the one hand, the advantage of these BRDF correction models is evident. However, all the existing models do not take mixed pixels into account. Ignoring the existence of mixed pixels while correcting the BRDF effect leads to undesired results. Thus, in this study, we address the influence of mixed pixels and propose an unmixing-based semi-empirical model for the BRDF correction while integrating the EMs' fraction within the process to estimate the weights of the BRDF kernels. Experiments under controlled laboratory conditions clearly show that the proposed methodology outperforms the traditional semi-empirical correction model in terms of generality and accuracy of the mixed pixels corrected signatures.

\section{METHODOLOGY}

One efficient correction of the BRDF effect relies on a semiempirical model that combines a sum of kernels to reconstruct an analytical function that describes the expected reflectance in each viewing direction. Two of these kernels represent geometric and volumetric scattering, while the third one 
characterizes isotropic reflectance. Current strategies rely on modulating the directional reflectance and correct the BRDF effect for each landcover type separately. Thus, we compute the modeled reflectance $\rho_{m}^{\prime}$ for a specific landcover type in each spectral band as follows:

$$
\begin{aligned}
& \rho_{m}^{\prime j}\left(\theta_{i}, \theta_{r}, \Delta \varphi\right)= \\
& f_{i s o}^{j}+f_{\text {vol }}^{j} \cdot k_{\text {vol }}\left(\theta_{i}, \theta_{r}, \Delta \varphi\right)+f_{\text {geo }}^{j} \cdot k_{\text {geo }}\left(\theta_{i}, \theta_{r}, \Delta \varphi\right)
\end{aligned}
$$

Where $\theta_{i}$ and $\theta_{r}$ are the solar and viewing zenith angles, respectively, and $\Delta \varphi$ is the phase angle. The superscript $j$ denotes the $j$-th class among several classes, i.e., landcover types, within the image. The notations $k_{v o l}, k_{\text {geo }}, f_{v o l}$, and $f_{\text {geo }}$ indicate the volumetric and geometric kernels and their weight coefficients, respectively, and $f_{i s o}$ is a scalar represents the isotropic reflectance. To create a semi-empirical model that fits for cases with high solar and(/or) view angles (Jia et al., 2020), we use the hotspot-revised Ross-Thick-Maignan (RTM) (Maignan et al., 2004) volumetric kernel and Li-TransitReciprocal (LTR) geometric kernel (Li et al., 1999; Zhang et al., 2018). The computation of the RTM and LTR kernels is given by:

$$
\begin{aligned}
& k_{v o l}=\frac{\left(\frac{\pi}{2}-\xi\right) \cos (\xi)+\sin (\xi)}{\cos \left(\theta_{i}\right)+\cos \left(\theta_{r}\right)}\left(1+\left(1+\frac{\xi}{\xi_{o}}\right)^{-1}\right)-\frac{\pi}{4}, \\
& \cos (\xi)=\cos \left(\theta_{i}\right) \cos \left(\theta_{r}\right)+\sin \left(\theta_{i}\right) \sin \left(\theta_{r}\right) \cos (\Delta \varphi)
\end{aligned}
$$

and

$$
\begin{gathered}
k_{\text {geo }}=\left\{\begin{array}{cc}
k_{\text {LiSparse }}, & B \leq 2 \\
k_{\text {LiDense }}=\frac{2}{B} k_{\text {LiSparse }}, & B>2 \\
k_{\text {LiSparse }}=
\end{array}\right. \\
O-\sec \left(\theta_{i}\right)-\sec \left(\theta_{r}\right)+\frac{1}{2}(1+\cos (\xi)) \sec \left(\theta_{r}\right) \sec \left(\theta_{i}\right) \\
B=\sec \left(\theta_{i}\right)+\sec \left(\theta_{r}\right)-O \\
O=\frac{1}{\pi}(t-\sin (t) \cos (t))\left(\sec \left(\theta_{i}\right)+\sec \left(\theta_{r}\right)\right) \\
\cos (t)=\frac{\sqrt{D^{2}+\left(\tan \left(\theta_{i}\right) \tan \left(\theta_{r}\right) \sin (\Delta \varphi)\right)^{2}}}{\sec \left(\theta_{i}\right)+\sec \left(\theta_{r}\right)} \\
D=\sqrt{\tan ^{2}\left(\theta_{i}\right)+\tan ^{2}\left(\theta_{r}\right)-2 \tan \left(\theta_{i}\right) \tan \left(\theta_{r}\right) \cos (\Delta \varphi)}
\end{gathered}
$$

Given the sun's and sensor's location, relative to the image pixel locations, the kernels' values are fixed for each pixel and calculated as presented in equations (2) and (3). Using pixels of the same landcover type but in different places over the images, i.e., with varying geometrical parameters, we can build the following system of linear equations:

$$
\left\{\begin{array}{l}
\rho_{1}=f_{i s o}+f_{v o l} \cdot k_{v o l, 1}+f_{g e o} \cdot k_{g e o, 1} \\
\rho_{2}=f_{i s o}+f_{v o l} \cdot k_{v o l, 2}+f_{g e o} \cdot k_{g e o, 2} \\
\vdots \\
\rho_{n}=f_{i s o}+f_{v o l} \cdot k_{v o l, n}+f_{g e o} \cdot k_{g e o, n}
\end{array}\right\}
$$

A least-squares solution of this equation system provides an estimate of the coefficients $f_{i s o}, f_{v o l}, f_{\text {geo }}$. We use these coefficients to calculate the anisotropy factor (Jia et al., 2020) for each measurement. The anisotropic factor describes the ratio between the directional reflectance and a reference reflectance. Usually, we correct the measurements to the nadir view, and the anisotropic factor for each pixel is then given by:

$$
\eta^{\prime}=\frac{\rho_{m}^{\prime}\left(\theta_{i}, \theta_{r}, \Delta \varphi\right)}{\rho_{m}^{\prime}\left(\theta_{i_{-} r e f}, \theta_{r_{-} r e f}, \Delta \varphi_{r_{-} r e f}\right)},
$$

where, $\theta_{i_{-} \text {ref }}, \theta_{r_{-} \text {ref }}$, and $\Delta \varphi_{r_{\text {ref }}}$ usually represent a nadir view reference direction, i.e., $\theta_{i \text { ref }}=0$. Then we correct the measured reflectance to reduce the BRDF effect as follows:

$$
\rho^{\prime}\left(\theta_{i}, \theta_{r}, \Delta \varphi\right)=\rho\left(\theta_{i}, \theta_{r}, \Delta \varphi\right) / \eta^{\prime},
$$

where $\rho, \quad \rho^{\prime}$ and $\eta^{\prime}$ are measured and corrected reflectance values, and the anisotropy factor according to the traditional semi-empirical model, respectively.

\subsection{An improved unmixing-based semi-empirical model for} BRDF correction

The semi-empirical approach for correcting the BRDF effect reduces the reflectance variability due to measuring from different directions. Although, two main drawbacks of the traditional BRDF correction models still existing: 1) the need for classifying the image into different landcover types, and 2) the nonconsideration of mixed pixels that contain more than one kind of material. Mixed pixels contain more than one material in different variations and different fraction compositions. Thus, it is highly probable that none of the estimated semi-empirical models for each material fits for correcting the BRDF of mixed pixels. Therefore, there is a need for an approach that takes these pixels into account. Here, we present a new modified semi-empirical model that considers the probable mixture within the pixel and does not require classifying the image into landcover types. Mainly, we propose an unmixing-based semiempirical model that allows for incorporating the EMs' fraction while estimating the model's kernel weights. Similar to the traditional model, we use the RTM and LTR kernels to compute $k_{v o l}$, and $k_{\text {geo }}$. Whereas, we apply the unmixing process to the data to estimate the EMs' fraction in each pixel

\subsection{Spectral Unmixing}

Assuming a linear mixture model, we represent the pixel's reflectance as a linear combination of the set of EMs as follows:

$$
\mathbf{r}=\mathbf{E} \mathbf{a}+\mathbf{w}
$$

where $\mathbf{r} \in \mathbb{R}^{b \times 1}$ is the pixel's reflectance, $\mathbf{E} \in \mathbb{R}^{b \times d}$ is a matrix of the EM spectra, $\mathbf{a}=\left[\alpha_{1}, \alpha_{2}, \cdots, \alpha_{d}\right]^{\mathrm{T}}$ is the fraction vector, and $\mathbf{w} \in \mathbb{R}^{b \times 1}$ represents the system noise and is assumed to be zero-mean Gaussian, while $b$ and $d$ are the number of spectral bands and number of EMs, respectively. To estimate the fraction vector, we solve an optimization problem to minimize an objective function that measures the spectral dissimilarity between the pixel's reflectance and the reconstructed reflectance by the EMs and their estimated fractions. Among various objective functions for the unmixing problem, the Euclidian 
Minimum Distance (EMD) is common. However, other measures, e.g., the Spectral Angle Mapper (SAM) (Kizel and Shoshany, 2018; Shoshany et al., 2011), are preferred for specific purposes, for example, reducing the varying illumination effect (Kizel et al., 2017). In addition, the unmixing problem is usually solved under constraints of positivity and additive sum-to-one over the pixel's fraction to ensure the physical feasibility of the results.

\subsection{A fraction-based estimation of BRDF coefficients}

Given the EM fraction in each pixel and assuming a linear mixture model, we represent the modeled reflectance as follows:

$$
\rho_{m}^{\prime \prime}=\alpha_{1} \rho_{m}^{\prime 1}+\alpha_{2} \rho_{m}^{\prime 2}+\cdots+\alpha_{2} \rho_{m}^{\prime d}
$$

Using the kernel-based formulation of the modeled reflectance of each landcover type, we can write the following

$$
\rho_{m}^{\prime \prime}=\sum_{j=1}^{d} \alpha_{j}\left(f_{i s o}^{j}+f_{v o l}^{j} \cdot k_{v o l}+f_{g e o}^{j} \cdot k_{g e o}\right) .
$$

Where $d$ is the number of different landcover types within the pixel and $\alpha_{1}, \alpha_{2}, \cdots, \alpha_{d}$ are their abundance fractions, which we estimate through an unmixing process (Kizel et al., 2017; Kizel and Shoshany, 2018). Similar to the case of the traditional semiempirical model, each pixel measurement provides one equation as follows:

$$
\left\{\begin{array}{l}
\rho_{1}=\sum_{j=1}^{d} \alpha_{j, 1}\left(f_{i s o}^{j}+f_{v o l}^{j} \cdot k_{v o l, 1}+f_{g e o}^{j} \cdot k_{g e o, 1}\right) \\
\rho_{2}=\sum_{j=1}^{d} \alpha_{j, 2}\left(f_{i s o}^{j}+f_{v o l}^{j} \cdot k_{v o l, 2}+f_{g e o}^{j} \cdot k_{g e o, 2}\right) \\
\vdots \\
\rho_{n}=\sum_{j=1}^{d} \alpha_{j, n}\left(f_{i s o}^{j}+f_{v o l}^{j} \cdot k_{v o l, n}+f_{g e o}^{j} \cdot k_{g e o, n}\right)
\end{array}\right\}
$$

Like in the classic model, solving this linear equation system, we estimate the coefficients $f_{i s o}, f_{v o l}, f_{g e o}$ for each landcover type, but simultaneously and without classifying the image. Once we estimate the model's coefficients, we then compute the corrected reflectance as

$$
\rho^{\prime \prime}\left(\theta_{i}, \theta_{r}, \Delta \varphi\right)=\rho\left(\theta_{i}, \theta_{r}, \Delta \varphi\right) / \eta^{\prime \prime}
$$

Where $\rho^{\prime \prime}$ and $\eta^{\prime \prime}$ are the corrected measurement and the computed anisotropy factor using the unmixing-based semiempirical model, respectively.

\section{EXPERIMENTS AND RESULTS}

To evaluate the proposed methodology, we experimented with real image data acquired under controlled conditions. We created BRDF scenarios using hand-crafted pixels with a microtopography by placing cubes made of one material on top of a homogenous background, made of another material (see Figure 1). Then, we acquired images of each scene from seven different camera locations while the light source was at a zenith angle $\theta_{i}=30^{\circ}$ and azimuth $\varphi_{i}=180^{\circ}$. Precisely, we located the camera at three solid angles: $\theta_{r}=0^{\circ}, 30^{\circ}, 60^{\circ}$ and three azimuth angles: $\varphi_{r}=0^{\circ}, 90^{\circ}, 270^{\circ}$ (see Figure 1). We used the Specim IQ hyperspectral camera for image acquisition, which provides images with a size of $512 \times 512$ pixels and 204 spectral bands between $400-1000 \mathrm{~nm}$. As a light source, we used the $\mathrm{C} 12$ silent halogen lamp provided by Hedler (https://www.hedler.com). We placed a barium sulfate calibration panel within the acquired frame, then retrieved the reflectance values in each image by dividing the spectral signature in each pixel by the calibration panel's mean signature. Then, to create mixed pixels, we aggregate each image into a single pixel. Once we have seven mixed pixels representing the same area but measured from different viewing locations, we apply the following steps to evaluate the new model:

1) Estimating the EM fractions in each pixel.

2) Estimating $f_{i s o}, f_{v o l}, f_{\text {geo }}$ for each EM (landcover type) according to the new model.

3) Correcting the reflectance value in each pixel using the estimated coefficients in step 2.

4) Calculate the coefficient of variation (CV) in each band over the different pixels.

We compute the CV in each spectral band as the ratio of the standard deviation $\sigma_{\lambda}$ to the mean $\mu_{\lambda}$ of the seven pixels as follows

$$
\mathrm{CV}_{\lambda}=100 \cdot \sigma_{\lambda} / \mu_{\lambda}
$$

where $\mu_{\lambda}=\frac{1}{n} \sum_{p=1}^{n} \rho_{p, \lambda}$ and $\sigma_{\lambda}=\sqrt{\frac{1}{n} \sum_{p=1}^{n}\left(\rho_{p, \lambda}-\mu_{\lambda}\right)^{2}}$ are the mean and standard deviation over the $n$ measured pixels at the spectral band denoted by $\lambda$, respectively, and $\rho_{p, \lambda}$ is the reflectance value at the $p$-th pixel. For comparison, we use the uncorrected measurements and those that we correct using the traditional model. Figure 2, Figure 3, and Figure 4 present the results for patterns $\mathrm{A}, \mathrm{B}$, and $\mathrm{C}$, respectively. In the original experiment, we tested six different patterns, but we present the results for only three patterns due to size limitations. The results clearly show that incorporating the EM fractions within a new semi-empirical model enhances the BRDF correction.

The advantage of the proposed unmixing-based model over the traditional model is apparent in all three cases (patterns). The plots of the measured and modeled reflectance values at a particular wavelength (first row in Figures 2-4) show how the proposed model captures the measured reflectance trend more accurately. Thus, the obtained corrected values are more uniform under the different viewing zenith angles. Both the traditional and proposed correction models reduce the BRDF effect's influence on the retrieved spectral signatures. But the $\mathrm{CV}$ values of the obtained corrected reflectance by the proposed unmixing-based model are lower than those obtained by the traditional model over the entire spectral bands. To derive more insights regarding the tested models' performance, we observe the mean value of the $\mathrm{CV}$ in each BRDF scenario. Table I summarizes the results that clearly show that the proposed unmixing-based model outperforms the traditional one. 

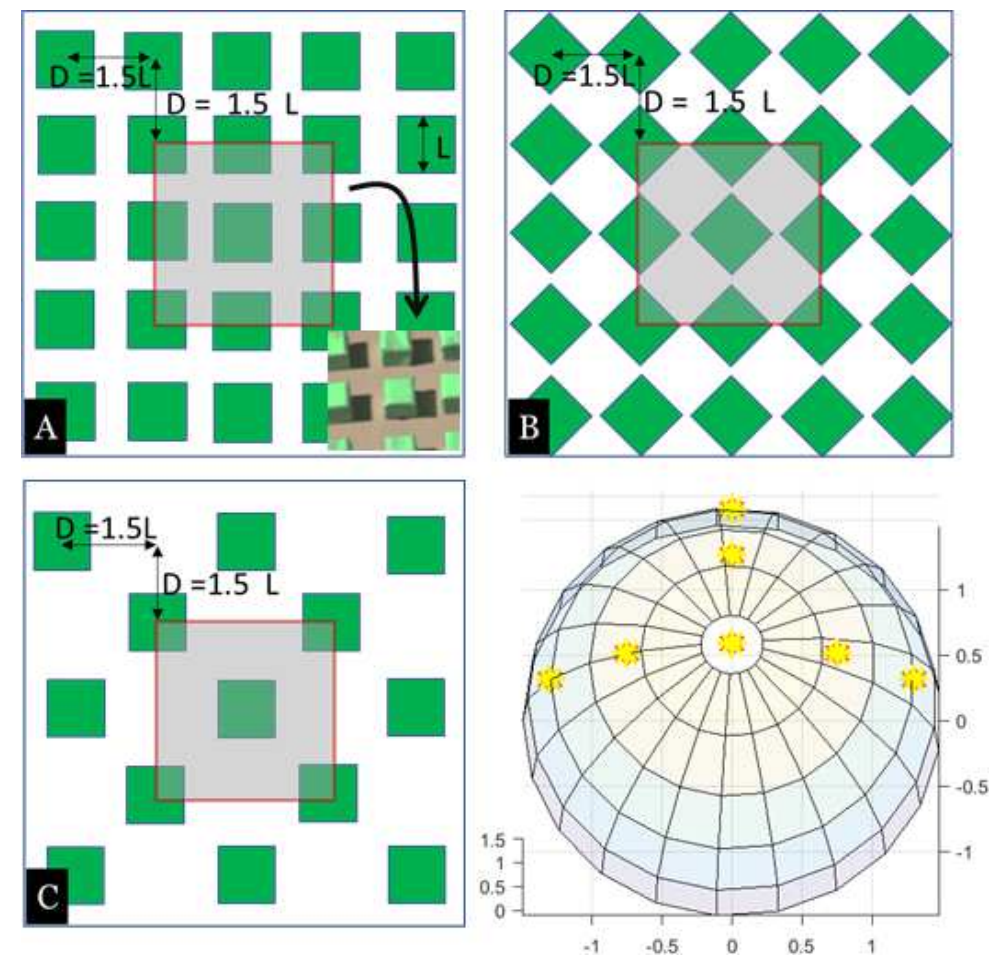

Figure 1. An illustration of the imaging configuration (righ bottom corner); the camera positions are marked on top of the hemisphere. Tiles A, B, and C show the repetitive patterns A, B, and C, respectively; the imaged area is marked with a square. This area is aggregated into a single pixel to create a spectral mixture. One of the real seven images for pattern A is shown in the right bottom corner of its corresponding tile.

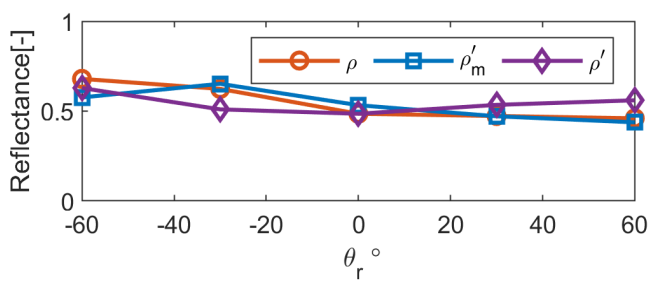

(a)

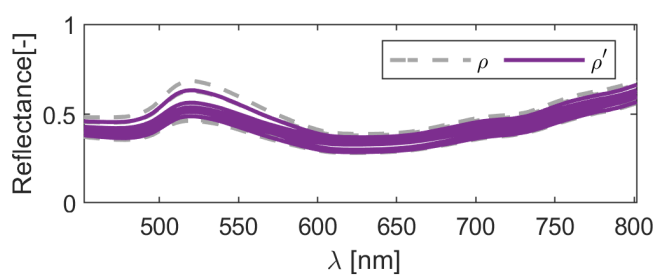

(c)

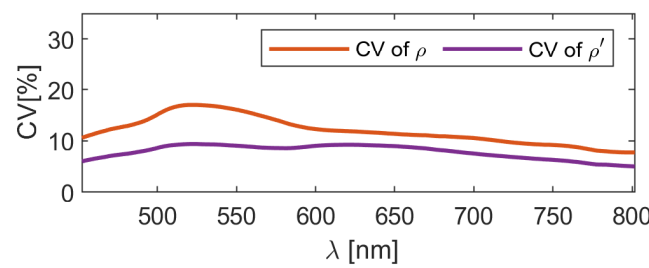

(e)

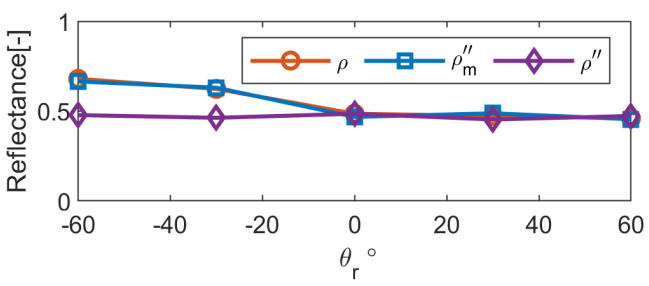

(b)

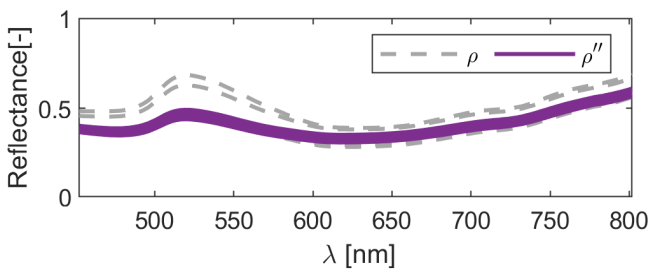

(d)

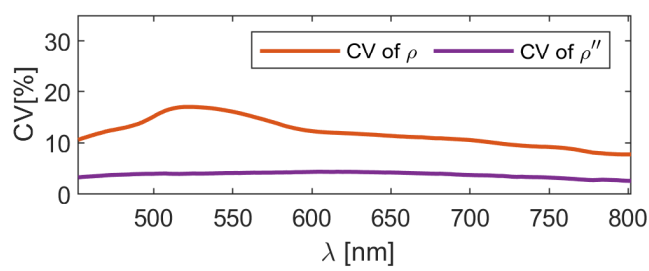

(f)

Figure 2. Evaluation of the BRDF correction models for the microstructure pattern A. Left and right columns, correction without considering and considering the spectral mixture, respectively. The second row presents the measured pixels' reflectance signatures before the BRDF correction (gray dashed lines) and after the correction (solid magenta lines). The third row shows the CV for each band before and after the correction. 


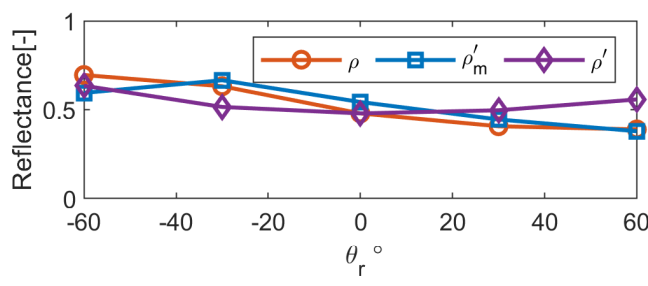

(a)

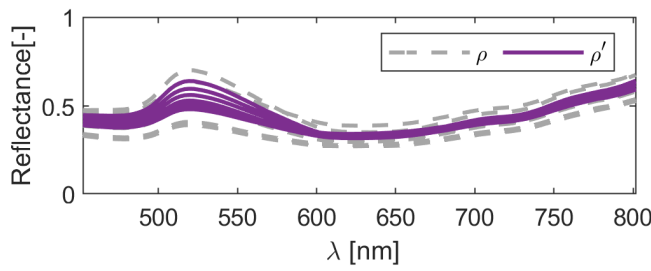

(c)

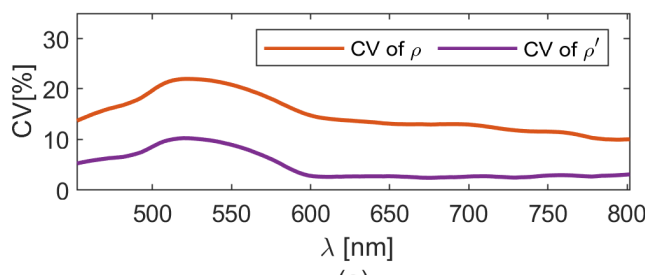

(e)

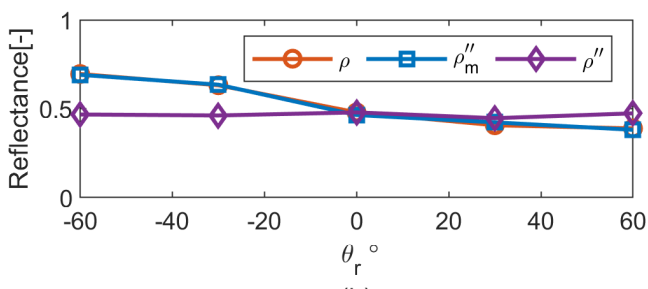

(b)

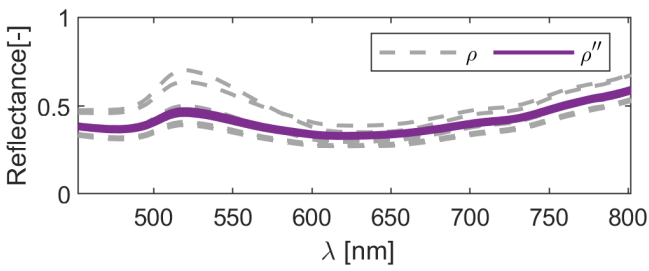

(d)

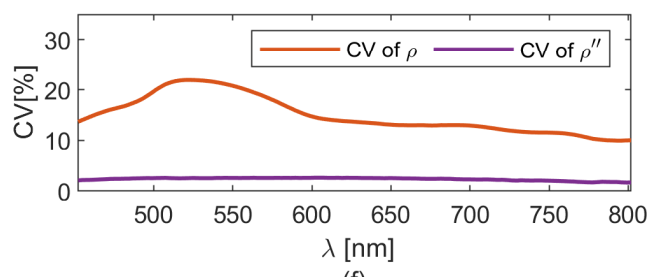

(f)

Figure 3. Evaluation of the BRDF correction models for the microstructure pattern B. Left and right columns, correction without considering and considering the spectral mixture, respectively. The second row presents the measured pixels' reflectance signatures before the BRDF correction (gray dashed lines) and after the correction (solid magenta lines). The third row shows the CV for each band before and after the correction.

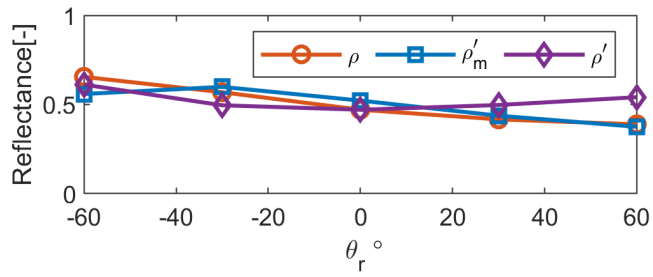

(a)

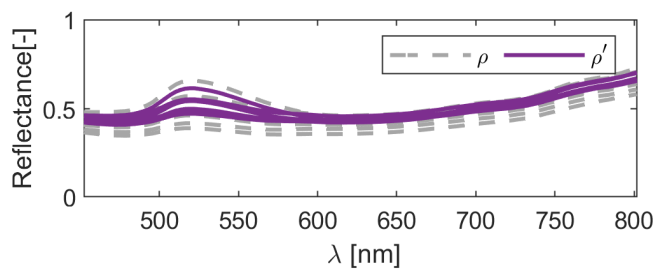

(c)

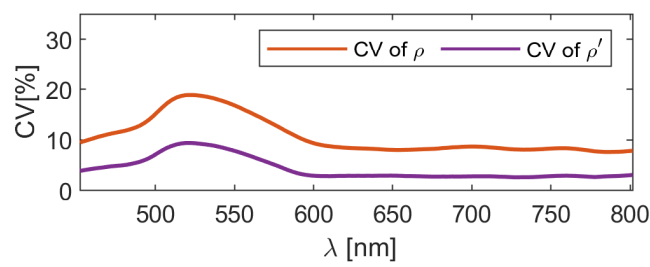

(e)

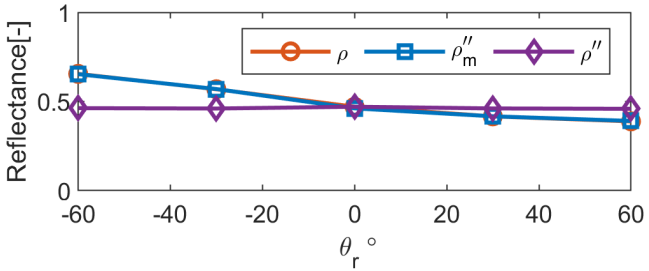

(b)

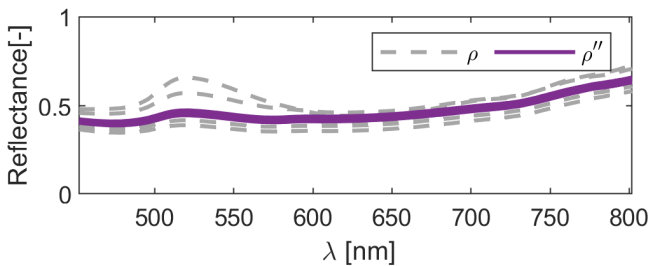

(d)

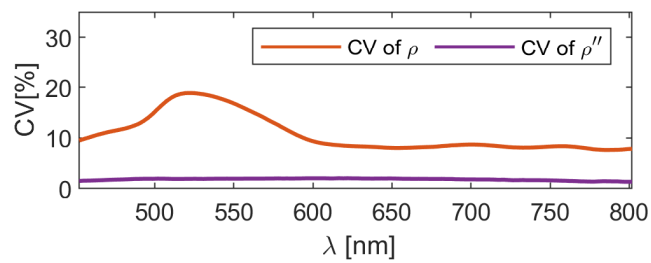

(f)

Figure 4. Evaluation of the BRDF correction models for the microstructure pattern C. Left and right columns, correction without considering and considering the spectral mixture, respectively. The second row presents the measured pixels' reflectance signatures before the BRDF correction (gray dashed lines) and after the correction (solid magenta lines). The third row shows the CV for each band before and after the correction. 
The performance of the unmixing-based model is advantageous regardless of the microtopography of the pattern. However, the comparison of the results for the different patterns is noteworthy. Despite using the same two EMs in all the cases, the $\mathrm{CV}$ of the corrected measurements varies from one pattern to another. This result shows the influence of the different shading portions due to the different microtopography and the variations of the cube facets orientation within the pattern.

\begin{tabular}{|l|c|c|c|}
\hline Pattern & $\rho$ & $\rho^{\prime}$ & $\rho^{\prime \prime}$ \\
\hline A & 12.0 & 7.9 & $\mathbf{3 . 8}$ \\
B & 15.0 & 4.7 & $\mathbf{2 . 4}$ \\
C & 10.8 & 4.3 & $\mathbf{1 . 8}$ \\
\hline
\end{tabular}

Table I. Summary of evaluation metrics CV of the measured and BRDF-corrected reflectance.

\section{CONCLUSIONS}

We proposed an unmixing-based model for correcting the BRDF effect in spectral data. The proposed model relies mainly on incorporating the abundance fractions of EMs within the regression problem for estimating the weights of BRDF kernels. We conducted an experimental evaluation with a mixture of two EMs and three different sub-pixel microstructure variations. The results indicate the BRDF correction's significant improvement through the unmixing-based model compared to the traditional semi-empirical one. The $\mathrm{CV}$ of the corrected data through the proposed model is significantly lower for all the examined patterns. In the meantime, we test the proposed model on a mixture of only two EMs, but the results are encouraging as a first step towards developing a new general model for the BRDF correction.

\section{REFERENCES}

Disney, M.J., Lewis, P., 1998. An investigation of how linear BRDF models deal with the complex scattering processes encountered in a real canopy, in: IGARSS '98. Sensing and Managing the Environment. 1998 IEEE International Geoscience and Remote Sensing. Symposium Proceedings. (Cat. No.98CH36174). IEEE, pp. 1231-1233 vol.3. https://doi.org/10.1109/IGARSS.1998.691360

Eismann, M.T., Meola, J., Hardie, R.C., 2008. Hyperspectral Change Detection in the Presenceof Diurnal and Seasonal Variations. IEEE Trans. Geosci. Remote Sens. 46, 237-249. https://doi.org/10.1109/TGRS.2007.907973

Faran, I., Netanyahu, N.S., Omid David, E., Shoshany, M., Kizel, F., Chang, J.G., Rud, R., 2019. Ground Truth Simulation for Deep Learning Classification of Mid-Resolution Venus Images Via Unmixing of High-Resolution Hyperspectral Fenix Data. https://doi.org/10.1109/igarss.2019.8900186

Jia, W., Pang, Y., Tortini, R., Schläpfer, D., Li, Z., Roujean, J.L., 2020. A kernel-driven BRDF approach to correct airborne hyperspectral imagery over forested areas with rugged topography. Remote Sens. https://doi.org/10.3390/rs12030432

Jiao, Z., Zhang, X., Bréon, F.M., Dong, Y., Schaaf, C.B., Román, M., Wang, Z., Cui, L., Yin, S., Ding, A., Wang, J., 2018. The influence of spatial resolution on the angular variation patterns of optical reflectance as retrieved from MODIS and POLDER measurements. Remote Sens. Environ. 215, 371-385. https://doi.org/10.1016/j.rse.2018.06.025

Keshava, N., Mustard, J.F., 2002. Spectral unmixing. IEEE Signal Process. Mag. 19, 44-57. https://doi.org/10.1109/79.974727

Kizel, F., Benediktsson, J.A., 2020. Spatially enhanced spectral unmixing through data fusion of spectral and visible images from different sensors. Remote Sens. 12, 1255. https://doi.org/10.3390/RS12081255

Kizel, F., Shoshany, M., 2018. Spatially adaptive hyperspectral unmixing through endmembers analytical localization based on sums of anisotropic 2D Gaussians. ISPRS J. Photogramm. Remote Sens. https://doi.org/10.1016/j.isprsjprs.2018.03.021

Kizel, F., Shoshany, M., Netanyahu, N.S., Even-Tzur, G., Benediktsson, J.A., 2017. A stepwise analytical projected gradient descent search for hyperspectral unmixing and its code vectorization. IEEE Trans. Geosci. Remote Sens. 55, 49254943. https://doi.org/10.1109/TGRS.2017.2692999

Landgrebe, D., 2002. Hyperspectral image data analysis. IEEE Signal Process. Mag. 19, 17-28. https://doi.org/10.1109/79.974718

Li, X., Gao, F., Chen, L., Strahler, A.H., 1999. Derivation and validation of a new kernel for kernel-driven BRDF models, in: Remote Sensing for Earth Science, Ocean, and Sea Ice Applications. https://doi.org/10.1117/12.373123

Maignan, F., Bréon, F.M., Lacaze, R., 2004. Bidirectional reflectance of Earth targets: Evaluation of analytical models using a large set of spaceborne measurements with emphasis on the Hot Spot. Remote Sens. Environ. https://doi.org/10.1016/j.rse.2003.12.006

Melgani, F., Bruzzone, L., 2004. Classification of hyperspectral remote sensing images with support vector machines. IEEE Trans. Geosci. Remote Sens. 42, 1778-1790. https://doi.org/10.1109/TGRS.2004.831865

Roberts, G., 2009. Progress in Physical Geography A review of the application of BRDF models to infer land cover parameters at regional and global scales. Prog. Phys. Geogr. 4, 483-511. https://doi.org/10.1177/030913330102500402

Roberts, G., 2001. A review of the application of BRDF models to infer land cover parameters at regional and global scales. Prog. Phys. Geogr. Earth Environ. 25, 483-511. https://doi.org/10.1177/030913330102500402

Roujean, J.L., Leroy, M., Dechamps, P., 1992. for the Correction of Remote Sensing Data. Techniques 97, 20-455.

Roy, D.P., Zhang, H.K., Ju, J., Gomez-Dans, J.L., Lewis, P.E., Schaaf, C.B., Sun, Q., Li, J., Huang, H., Kovalskyy, V., 2016. A general method to normalize Landsat reflectance data to nadir BRDF adjusted reflectance. Remote Sens. Environ. 176, 255271. https://doi.org/10.1016/j.rse.2016.01.023

Shaw, G.A., Burke, H.K., 2003. Spectral Imaging for Remote Sensing. LINCOLN Lab. J. 14, 3-28. https://doi.org/10.1108/14777830310460360

Shoshany, M., Kizel, F., Netanyahu, N.S., Goldshlager, N., Jarmer, T., Even-Tzur, G., 2011. An iterative search in endmember fraction space for spectral unmixing. IEEE Geosci. Remote Sens. Lett. 8, 706-709. https://doi.org/10.1109/LGRS.2010.2101578

Walthall, C.L., 1997. A study of reflectance anisotropy and canopy structure using a simple empirical model. Remote Sens. Environ. 61, 118-128. https://doi.org/10.1016/S00344257(96)00245-3

Zhang, X., Jiao, Z., Dong, Y., Zhang, H., Li, Y., He, D., Ding, A., Yin, S., Cui, L., Chang, Y., 2018. Potential investigation of linking PROSAIL with the Ross-Li BRDF model for vegetation characterization. https://doi.org/10.3390/rs10030437 